\title{
Inflammatory and coagulatory parameters linked to survival in critically ill children with sepsis
}

\author{
Christian Niederwanger ${ }^{1}$, Mirjam Bachler ${ }^{2}$, Tobias Hell ${ }^{3}$, Caroline Linhart ${ }^{4}$, Andreas Entenmann ${ }^{1}$, Agnes Balog $^{1}$, \\ Katharina Auer ${ }^{5}$ and Petra Innerhofer ${ }^{6}$
}

\begin{abstract}
Background: Sepsis is associated with a deflection of inflammatory and coagulative parameters, since some clotting factors are known to be involved in the host's defense against infection and inflammation. These parameters could play a crucial role in the course of sepsis and be used as prognostic markers in critically ill children.

Methods: A total of 250 critically ill pediatric patients diagnosed with sepsis were retrospectively analyzed to identify routinely measured predictors for in-hospital mortality at the peak level of C-reactive protein. Those parameters entered multivariate logistic regression analysis as well as a decision tree for survival.

Results: Multivariate logistic regression analysis revealed fibrinogen, platelets and activated partial thromboplastin time (aPTT) at the peak level of C-reactive protein to be predictors for survival $(p=0.03, p=0.01$ and $p=0.02$, respectively). An increase in fibrinogen and platelets is linked to survival, whereas an aPTT prolongation is associated with higher mortality; adjusted odds ratios (95\% Cl) for an increase of $100 \mathrm{mg} / \mathrm{dl}$ in fibrinogen are 1.35 (1.04-1.82) per $50 \mathrm{G} / \mathrm{l}$ platelets $1.94(1.3-3.29)$ and $0.83(0.69-0.96)$ for an aPTT prolongation of $10 \mathrm{~s}$. Decision tree analysis shows that a fibrinogen level below $192 \mathrm{mg} / \mathrm{dl}$ (90.9\% vs. 13\% mortality) is most distinctive in non-survivors.

Conclusions: High levels of fibrinogen and platelets as well as a non-overshooting aPTT are associated with a higher survival rate in pediatric patients with diagnosed sepsis. In particular, hypofibrinogenemia is distinctive for a high mortality rate in septic critically ill children.
\end{abstract}

Keywords: Fibrinogen, Sepsis, Children, Survival, Inflammation, Coagulation, Platelets

\section{Introduction}

Although the number of deaths caused by sepsis has drastically decreased in the last couple of decades [1], sepsis remains one of the main causes of mortality in infants and toddlers worldwide [1-3]. Sepsis in children peaks in the neonatal period and symptoms may be nonspecific in those patients [4], while older children may show hyperthermia, tachycardia, tachypnea, hypotension

\footnotetext{
*Correspondence: mirjam.bachler@tirol-kliniken.at

${ }^{2}$ Institute for Sports Medicine, Alpine Medicine and Health Tourism, UMIT - University for Health Sciences, Medical Informatics and Technology, Eduard Wallnöfer Zentrum 1, 6060 Hall in Tirol, Austria

Full list of author information is available at the end of the article
}

and disorders in hemostasis up to the clinical picture of disseminated intravascular coagulation (DIC).

Sepsis is initially characterized by excessive production of pro-inflammatory cytokines, leukocyte activation and tissue damage, followed by release of anti-inflammatory cytokines, leukocyte deactivation and immunosuppression [5]. In the later phase of sepsis, compensatory release of anti-inflammatory molecules is thought to mediate a state of immunosuppression associated with significant impairment of immune cell function (immunoparalysis) [6].

The systemic inflammation during sepsis is observed by measuring leukocytes, procalcitonin, $\mathrm{C}$-reactive protein and others. Leukocyte count is part of the definition of sepsis, but only at extreme levels are leukocytes 
associated with the progression of sepsis [7]. Data confirm that procalcitonin, which specifically increases in bacterial processes [8], is suitable as a diagnostic parameter in many cases in adults due to its high specificity $[9,10]$. However, limited data are available for use in pediatric patients [11]. C-reactive protein reflects the inflammatory process and is widely used in clinical routine. Many studies have described an interrelation between an elevated C-reactive protein level and sepsis [12-15]. Thus, in clinical routine, daily C-reactive protein measurements might be used to assess the efficacy of treatment [16]. C-reactive protein can also be an indicator of organ failure [17-21] and therefore has potential for the surveillance of sepsis severity.

Systemic inflammation is followed by activation of the coagulation system, and conversely, components of the coagulation system significantly affect the inflammatory response [22]. This cross-link occurs on several levels: Pro-inflammatory cytokines stimulate the production of coagulation factors and coagulation factors mediate inflammation via binding to receptors of endothelium and immune cells [23]. The coagulation factors also play a direct role in host defense. For example, fibrinogen modulates the immune system [24, 25] and acts as a chemotactical factor for monocytes and neutrophils [26]. Furthermore, as part of the innate immune system, it limits bacterial spreading by forming fiber nets in which the pathogens become immobilized [24].

With few exceptions, such as antithrombin or platelets, the role of coagulation parameters in sepsis is largely ignored. New diagnostic, prognostic and therapeutic strategies can be deduced by observing coagulating laboratory data and, where appropriate, modifying them in the event of excessive activation or dysregulation of the system $[27,28]$. An increase in the pro-coagulatory parameters to high level is seen rather negatively in the inflammatory process because of their pro-thrombotic potential [29], although elevation of the pro-coagulatory parameters might be beneficial during sepsis due to their role in host defense.

Therefore, it is worthwhile to examine the behavior and influence of coagulation parameters in combination with the typical inflammatory parameters during sepsis. Correct interpretation of these could facilitate assessment of the course of sepsis in critically ill children.

\section{Methods}

This retrospective analysis comprises clinical data and routine laboratory parameters from 250 pediatric patients at the Pediatric Intensive Care Unit of Innsbruck Medical University Hospital.

\section{Inclusion of patients}

All medical files of patients younger than 18 years of age who were treated at the pediatric intensive care unit (PICU) between January 1, 2000, and December 31, 2014, with the diagnosis of sepsis or systemic infection were reviewed. A total of 250 patients met the sepsis criteria of Goldstein [1]. There was no need to obtain oral and written informed consent from the study participants since the data were processed anonymously. The study protocol was approved by the institutional review board of the Medical University of Innsbruck (AN2013-0044).

\section{Data collection}

We collected the demographic variables age, sex and the diagnosed underlying disease that triggered hospitalization. Septic shock was defined as the need for a vasoactive drug to maintain blood pressure in the normal range during the septic episode [1]. The C-reactive protein level was used to objectify the progression of sepsis, because it is an established parameter of sepsis [12-16] and is used in our clinic in children of all ages as opposed to IL- 6 and procalcitonin.

The day on which $\mathrm{C}$-reactive protein peaked was defined as day 0 in the report. The available routine parameters (fibrinogen, platelets, antithrombin, PT, aPTT, leukocytes) were observed from 3 days before until 3 days after day 0 . In-hospital mortality was chosen as the outcome parameter.

To evaluate changes in therapy and outcome due to the protracted study period, we grouped patients into four time cohorts (2000-2004, 2005-2007, 2008-2010 and 2011-2014).

\section{Statistical analysis}

A mathematician not involved in the study procedures or patient assessment $(\mathrm{TH})$ was responsible for the statistical analyses using $\mathrm{R}$, version 3.4.1. All statistical assessments were two sided, and a significance level of $5 \%$ was used. The hypothesis of a normal distribution was not reasonable for most of the continuous variables (Shapiro-Wilk normality test). The Wilcoxon rank sum test and Fisher's exact test were applied to assess differences between survivors and non-survivors. We present continuous data as medians (25th-75th percentile) and binary variables as no./total no. (\%). We show effect size and precision with estimated median differences between survivors and non-survivors for continuous data and odds ratios (OR) for binary variables, with 95\% CIs.

Stratified by survival, the progression of inflammation- and coagulation-related parameters from 3 days 
prior to 3 days after the peak of C-reactive protein is illustrated by the sequence of the median with corresponding $95 \%$ CIs in a purely descriptive manner.

In the univariate analysis, significant predictors at the peak of C-reactive protein as well as the respective patient's characteristics for survival were identified. Those variables entered a forward-backward stepwise selection by Akaike information criterion (AIC) to fit a logistic regression model for survival [30]. We provide adjusted odds ratios for the remaining predictors with 95\% CIs. Receiver operating characteristic (ROC curves) analysis was performed, and ROC AUC is provided with 95\% CIs. In addition, with the variables that entered the stepwise model selection, a recursive partitioning tree for survival was fitted using the R package rpart (version 4.111). All analyses were performed in a purely exploratory fashion.

\section{Results}

\section{Patient characteristics}

In total, 250 patients met the eligibility criteria for study inclusion and final analysis. Of those septic children, 41 (16.4\%) did not survive while in hospital. Of the critically ill children, $53.2 \%$ suffered from sepsis and $26.4 \%$ from severe sepsis. Septic shock was reported in 51/250 (20.4\%) children, of whom 21/51 (41.2\%) died. Patients' baseline characteristics stratified for survival and nonsurvival are presented in Table 1.

The population consisted of $134 / 250$ (53.6\%) male patients, and median (25th-75th percentile) age was 35
(6-109) months. The 22/249 (8.8\%) neonates showed a significantly higher $(p=0.02)$ mortality rate, because $8 / 22(36.4 \%)$ neonates as compared to $33 / 227$ (14.5\%) children older than 1 month did not survive the septic episode. The most commonly affected organ systems resulting in ICU admission were the respiratory system in $51 / 250(20.4 \%)$ and the central nervous system in $46 / 250$ (18.4\%) children.

To rule out possible confounding due to the protracted study period (2000-2014), we grouped patients into four time cohorts: 80 patients were included in 2000-2004, 56 in 2005-2007, 68 in 2008-2010 and 46 in $2011-2014$. Mortality was $12.5 \%, 28.6 \%, 13.2 \%$ and $13 \%$, respectively, and was not significantly associated with the time periods (Fisher's exact test: $p=0.07$ ).

\section{Univariate analysis of parameters at the peak level of C-reactive protein}

As presented in Table 2, no significant difference was observed in C-reactive protein or leukocyte levels between survivors and non-survivors at the peak level of C-reactive protein. There was a difference in the timing between hospital admission and C-reactive protein peak between survivors and non-survivors $(p<0.0001)$ with non-survivors having a longer time period to reach the $\mathrm{C}$-reactive protein peak. In contrast, fibrinogen, platelets and antithrombin significantly differ. This is also the case for aPTT and PT.

Table 1 Characteristics of patients stratified for survival and non-survival

\begin{tabular}{|c|c|c|c|c|c|}
\hline Characteristics $^{a}$ & Total $(n=250)$ & Survivors $(n=209)$ & Non-survivors $(n=41)$ & Estimate with $95 \% \mathrm{Cl}^{\mathrm{b}}$ & $p$ value $^{c}$ \\
\hline Female gender & $116 / 250(46.4 \%)$ & $97 / 209$ (46.4\%) & $19 / 41(46.3 \%)$ & $1(0.48$ to 2.06$)$ & 1 \\
\hline Age $^{\mathrm{d}}$ (months) & $34.83(6.47-108.63)$ & $35.5(7.16-111.45)$ & $23.8(1.43-81.97)$ & $3.33(-6.55$ to 18.07$)$ & 0.2725 \\
\hline Neonates < 1 month & $22 / 249(8.8 \%)$ & $14 / 208(6.7 \%)$ & $8 / 41(19.5 \%)$ & 3.34 (1.12 to 9.34$)$ & 0.0149 \\
\hline Infants $1-3$ months & $24 / 249(9.6 \%)$ & 20/208 (9.6\%) & $4 / 41(9.8 \%)$ & 1.02 (0.24 to 3.28$)$ & 1 \\
\hline \multicolumn{6}{|c|}{ Diagnosed underlying disease } \\
\hline Central nervous system & $46 / 250(18.4 \%)$ & $34 / 209$ (16.3\%) & $12 / 41(29.3 \%)$ & 2.12 (0.89 to 4.82) & 0.075 \\
\hline Cardiovascular & $39 / 250(15.6 \%)$ & $31 / 209(14.8 \%)$ & $8 / 41(19.5 \%)$ & 1.39 (0.51 to 3.45$)$ & 0.4808 \\
\hline Digestive tract & $41 / 250(16.4 \%)$ & $32 / 209(15.3 \%)$ & $9 / 41(22 \%)$ & 1.55 (0.59 to 3.74$)$ & 0.3546 \\
\hline Respiratory system & $51 / 250(20.4 \%)$ & $41 / 209$ (19.6\%) & $10 / 41(24.4 \%)$ & 1.32 (0.53 to 3.05$)$ & 0.5258 \\
\hline Oncologic & $37 / 250(14.8 \%)$ & $31 / 209$ (14.8\%) & $6 / 41(14.6 \%)$ & 0.98 (0.31 to 2.64) & 1 \\
\hline Kidney & $30 / 250$ (12\%) & 22/209 (10.5\%) & 8/41 (19.5\%) & 2.05 (0.73 to 5.31) & 0.1169 \\
\hline Liver & $18 / 250(7.2 \%)$ & 15/209 (7.2\%) & $3 / 41(7.3 \%)$ & 1.02 (0.18 to 3.87) & 1 \\
\hline Skin & $11 / 250(4.4 \%)$ & 10/209 (4.8\%) & $1 / 41(2.4 \%)$ & 0.5 (0.01 to 3.68) & 1 \\
\hline Other diagnosis & $30 / 250$ (12\%) & $25 / 209$ (12\%) & $5 / 41(12.2 \%)$ & 1.02 (0.29 to 2.97) & 1 \\
\hline
\end{tabular}

\footnotetext{
a Binary data are presented as no./total no. (\%) and continuous data as medians (25th-75th percentile)

b Odds ratio for binary variables and estimated median difference for continuous variables

c Differences between survivors and non-survivors assessed with Fisher's exact test for binary variables and Wilcoxon rank sum test for continuous variables

d For one survivor, the exact age in months is not known
} 
Table 2 Parameters at the peak level of C-reactive protein stratified for survival and non-survival

\begin{tabular}{|c|c|c|c|c|c|c|}
\hline Parameters $^{\mathbf{a}}$ & Total $(n=250)$ & Survivors $(n=209)$ & Non-survivors $(n=41)$ & Estimate with $95 \% \mathrm{Cl}^{\mathbf{b}}$ & $p$ value $^{c}$ & Not known ${ }^{d}$ \\
\hline C-reactive protein (mg/dl) & $18.5(9.8-28.4)$ & $18.6(10-28)$ & $17.6(7.9-31.9)$ & $-0.13(-5.3$ to 4.55$)$ & 0.9491 & $0 / 0$ \\
\hline Fibrinogen (mg/dl) & $506(329-675)$ & $518(383-685)$ & $279(173-514)$ & 206 (118 to 289) & $<0.0001$ & $56 / 9$ \\
\hline Platelets (G/I) & $124(50-232)$ & $147(62.5-247)$ & $63(29-98)$ & $74(37$ to 111$)$ & $<0.0001$ & $5 / 0$ \\
\hline aPTT (s) & $47(38-56)$ & $46(36-53)$ & $64(53.5-83)$ & $-20(-28$ to -13$)$ & $<0.0001$ & $59 / 15$ \\
\hline PT (\%) & $71(51-84)$ & $74(55-85)$ & $49(36-71.5)$ & 20 (10 to 30$)$ & 0.0001 & $59 / 14$ \\
\hline Antithrombin (\%) & $63(47-81)$ & $65.5(50-82)$ & $51(40-66)$ & $14(5$ to 23$)$ & 0.0045 & $75 / 10$ \\
\hline Leukocytes (G/I) & $10.4(4.6-17.8)$ & $11.1(5.3-17.5)$ & $8.2(3-20)$ & $1.4(-1.6$ to 5.1$)$ & 0.3342 & $4 / 5$ \\
\hline
\end{tabular}

a Data are presented as medians (25th-75th percentile)

b Estimated median difference

c Differences between survivors and non-survivors assessed with Wilcoxon rank sum test for continuous variables

d Number of missing measurements in survivors/non-survivors

\section{Progression of parameters around the peak level of C-reactive protein}

The course of fibrinogen and antithrombin seems to be associated with the progression of C-reactive protein (Figs. 1, 2). Fibrinogen concentration followed the progression of C-reactive protein in survivors, but not in non-survivors. Note that for the main portion of the survivors, fibrinogen levels increased distinctly above the norm values around day 0 .

Antithrombin levels were constantly higher in survivors than in non-survivors, but, in contrast to fibrinogen, irrespective of the progression of C-reactive protein. In survivors, platelets decreased slightly over time, while patients who did not survive showed a sharp decline in platelets already 2 days before the peak level of C-reactive protein was reached. Survivors showed significantly higher PT levels and shorter aPTT around the peak of C-reactive protein. Progression of leukocytes between survivors and non-survivors is comparable up to day 2 .

CRP, fibrinogen, platelets, aPTT, PT and antithrombin levels were comparable between time cohorts (KruskalWallis test: $p=0.40, p=0.32, p=0.58, p=0.90, p=0.17$ and $p=0.22$, respectively). Leukocyte count significantly differed between time cohorts (Kruskal-Wallis test: $p=0.04)$. Median leukocyte counts in $\mathrm{G} / \mathrm{l}$ were $12.3(6.13-20)$ in $2000-2004,12.1(6.8-17.1)$ in 2005$2007,10.3(3.1-16.4)$ in $2008-2010$ and $7.7(2.8-12)$ in 2011-2014.

\section{Multivariate logistic regression analysis for survival}

The following significant univariate predictors for survival entered a stepwise model selection: fibrinogen, platelets, antithrombin, aPTT, PT and age. The resulting logistic regression model for survival includes fibrinogen, platelets and aPTT as variables, all of them being significant predictors for survival $(p=0.03, p=0.01$ and $p=0.02$, respectively).
Figure 3 shows the adjusted odds ratios $(95 \% \mathrm{CI})$ retrieved from logistic regression. An increase in fibrinogen and platelets is linked to survival, whereas aPTT prolongation is associated with higher mortality. An increase of $100 \mathrm{mg} / \mathrm{dl}$ in fibrinogen increases the survival chance by $26 \%$, per $50 \mathrm{G} / 1$ platelets by $48.4 \%$, and aPTT prolongation of $10 \mathrm{~s}$ increases the mortality risk by $20.8 \%$.

Adding the time cohort or the year of CRP peak as input variables resulted in the identical logistic regression model after stepwise model selection.

The ROC analysis (Additional file 1) for survival predicted by fibrinogen, platelets and aPTT resulted in an AUC of 0.74 (0.63-0.85), $0.71(0.63-0.79)$ and 0.81 (0.73$0.90)$, respectively.

\section{Decision tree for survival}

To gain a deeper insight into the interplay of coagulation parameters leading to death in septic children, a decision tree was fitted using the univariate predictors at the peak level of C-reactive protein and age to explore these interrelations.

The obtained classification tree (see Fig. 4) shows that most distinctive in non-survivors is a fibrinogen level below $192 \mathrm{mg} / \mathrm{dl}$ [90.9\% (58.7-99.8\%) vs. 13\% (8.1$18.5 \%)$ mortality]. For patients presenting with a fibrinogen level of at least $192 \mathrm{mg} / \mathrm{dl}$, an aPTT of less than $58 \mathrm{~s}$ is associated with better outcome [8.4\% (5.0-13.1\%) vs. $37.8 \%(22.5-55.2 \%)$ mortality]. If, in addition, patients show a platelet count of at least $80 \mathrm{G} / \mathrm{l}$, a low mortality rate is observed $[4.1 \%(1.5-8.7 \%)$ vs. $20 \%(10.4-33.0 \%)$ mortality]. Patients with a platelet count below 80 G/l and younger than 1.2 months show high mortality as compared to older [57.1\% (18.4-90.1\%) vs. $14.6 \%$ $(6.1-27.8 \%)]$.

In the case of a fibrinogen level of at least $192 \mathrm{mg} / \mathrm{dl}$ and an aPTT prolongation of $58 \mathrm{~s}$ or more, antithrombin levels below $58 \%$ led to lower mortality [25\% (9.8-46.7\%) 


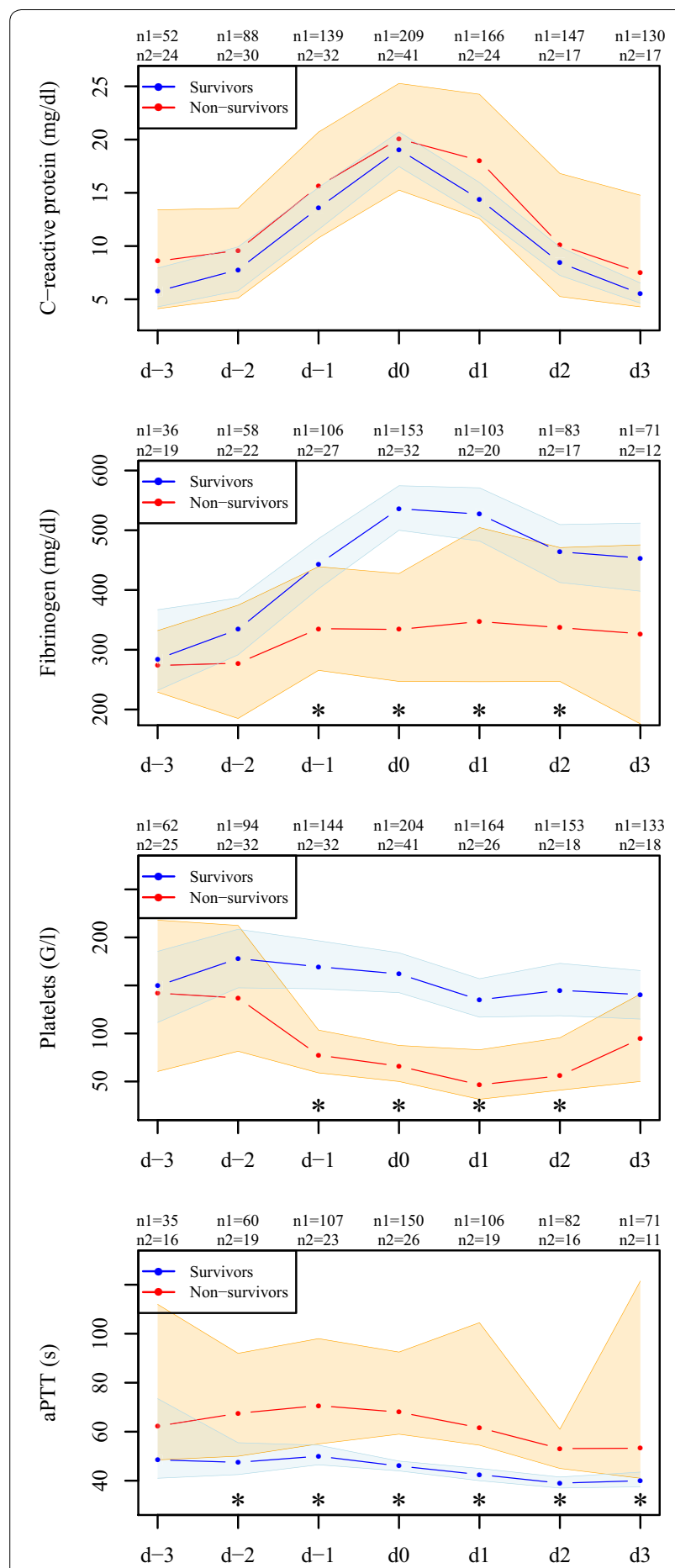

Fig. 1 Progression of C-reactive protein and measured coagulation parameters. Depicted are medians with $95 \% \mathrm{Cls}$ of the measured parameters for survivors (blue) and non-survivors (red) from 3 days prior to ( $d$-3 to $d-1$ ) until 3 days after ( $d 1$ to $d 3$ ) C-reactive protein peaked $(\mathrm{d} 0)$. Asterisks indicate significant differences between survivors and non-survivors at the respective time point; $n 1$ is the number of available measurements for survivors and $n 2$ for non-survivors vs. $61.5 \%(31.6-86.1 \%)]$, especially in patients exceeding an aPTT of $66 \mathrm{~s}$ [0\% (0.0-24.7\%) vs. $54.5 \%(23.4-83.3 \%)$ mortality].

The identical decision tree was obtained when the time cohort or the year of $\mathrm{C}$-reactive protein peak was added as predictors for survival, indicating that there was no confounding due to the protracted study period (2000-2014).

In order to predict mortality with fibrinogen $<192 \mathrm{mg} /$ $\mathrm{dl}$, platelets $<80 \mathrm{G} / \mathrm{l}$ and aPTT $>58 \mathrm{~s}$, sensitivity and specificity were calculated (Additional file 2).

\section{Age-adjusted hypofibrinogenemia}

The fibrinogen level of each child was classified as hypo-, normo- or hyperfibrinogenemia according to the agedependent norm value ranges. For normal fibrinogen values, please see the table in Additional file 3.

Patients presenting with hypofibrinogenemia have a significantly higher mortality rate than do patients with normo- or hyperfibrinogenemia: OR 28.42 (5.42-284.81), $p<0.0001$. As depicted in Fig. 5, 82\% of the patients with low fibrinogen levels died, whereas the mortality rate in patients with fibrinogen levels within the normal range was $22 \%$ and $10 \%$ in children with an elevated fibrinogen level.

No significant difference in the occurrence of a diagnosed thromboembolic event was observed for the 5/128 (3.9\%) children with hyperfibrinogenemia as compared to the $4 / 56(7.1 \%)$ patients with fibrinogen within the normal values or below: OR $0.53(0.11-2.78), p=0.46$.

\section{Discussion}

In this study, we investigated the impact of routinely measured coagulation and inflammation parameters on in-hospital survival in pediatric patients diagnosed with sepsis. The main result is that $\mathrm{C}$-reactive protein at its peak level does not significantly differ between survivors and non-survivors, whereas fibrinogen, platelets and aPTT are predictors of survival. An increase in fibrinogen and platelets is linked to survival, whereas aPTT prolongation is associated with higher mortality. Most distinctive for mortality are fibrinogen levels below $192 \mathrm{mg} / \mathrm{dl}$. Especially in children younger than 1.2 months, isolated thrombocytopenia is associated with poor outcome. In general, age-adjusted hypofibrinogenemia is significantly associated with a poor chance of survival.

In some points, these results stand in contrast to findings reported in the literature. Several studies claim that C-reactive protein is of prognostic value in sepsis [12-15]. However, in our study focusing on mortality, C-reactive protein levels did not allow any differentiation between survivors and non-survivors during the observation period of 7 days. Most likely, in children as 


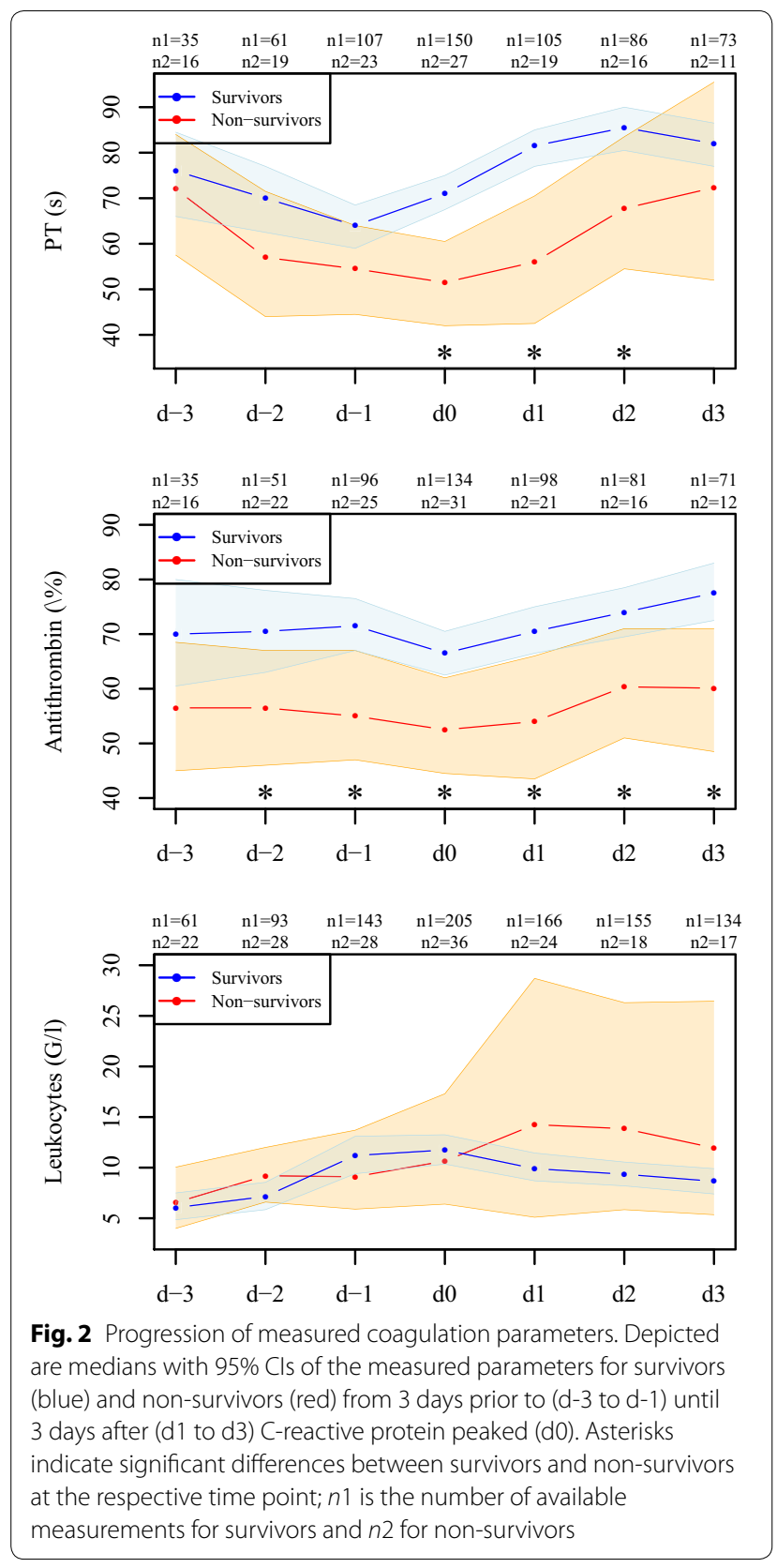

well as in adults with diagnosed sepsis C-reactive protein levels are highly elevated without predicting final outcome.

Among the variables routinely measured in our study at the peak level of $\mathrm{C}$-reactive protein, multivariate analysis revealed that only three of these coagulation parameters are essential for the prediction of survival in septic children: An increase in fibrinogen and platelets lowers the mortality risk, while a prolongation of aPTT increases the risk.
We found that hypofibrinogenemia is associated with very high mortality. This is in accordance with Esroy et al., who also confirm in their study that deceased patients exhibited significantly lower fibrinogen levels than did survivors [31]. Moreover, in baboons, survivors had higher fibrinogen levels than did non-survivors after induced sepsis [32]. This was also found in several earlier studies with fibrinogen-deficient mice following induced sepsis [33, 34].

The reason why fibrinogen is associated with survival in sepsis could be that it helps the immune system limit bacterial growth and enhance bacterial clearance [35]. The fibrin net captures and immobilizes invasive bacteria [24], thus restricting local spreading [24, 36]. Once the fibrinolysis sets in, plasminogen releases fibrinogen-derived so-called antimicrobial peptides (AMPs), thus causing an antimicrobial environment to arise within the clot. Such a peptide is the Bß15-42 fragment, and an unambiguous antimicrobial effect of this protein was already proven by $S$. aureus, group A Streptococcus (GAS) and group B Streptococcus (GBS) [25]. Besides that, this peptide binds to the VE cadherin of the endothelial cells and thus reinforces the tight junctions, which has a positive effect on organ failure and survival during sepsis $[37,38]$. The importance of fibrinogen, fibrinolysis and the consequently released peptides during sepsis and their beneficial impact on infection, multiple-organ dysfunction and reduced mortality have already been proven in several studies [39-41].

In our study, age-adjusted hyperfibrinogenemia is associated with increased survival in sepsis. In studies of adult patients, this connection between acute and transient fibrinogen levels in the context of sepsis and survival was also observed [42]. In this context of high levels of pro-thrombotic factors, the fear of a hypercoagulatory state during sepsis is present and justified, since this could contribute to the development of thrombosis. In our study, the incidence rate of thromboembolic events was not significantly increased in patients with hyperfibrinogenemia.

Low fibrinogen levels may reflect ongoing consumption and deposition, development of DIC and MODS [43, 44]. Sepsis itself also causes severe damage to the liver, via hemodynamic changes as well as the direct or indirect destruction of hepatocytes or both [45]. The destroyed hepatocytes are no longer able to synthesize a sufficient amount of fibrinogen, which might be another reason for the low fibrinogen levels in non-survivors.

High concentrations of fibrinogen before the C-reactive protein peak in our study rarely coincided with platelet concentrations below the critical limit of $100 \mathrm{G} / \mathrm{l}$. Low platelets in addition to low fibrinogen levels result in weak clot firmness, which is known to lead 


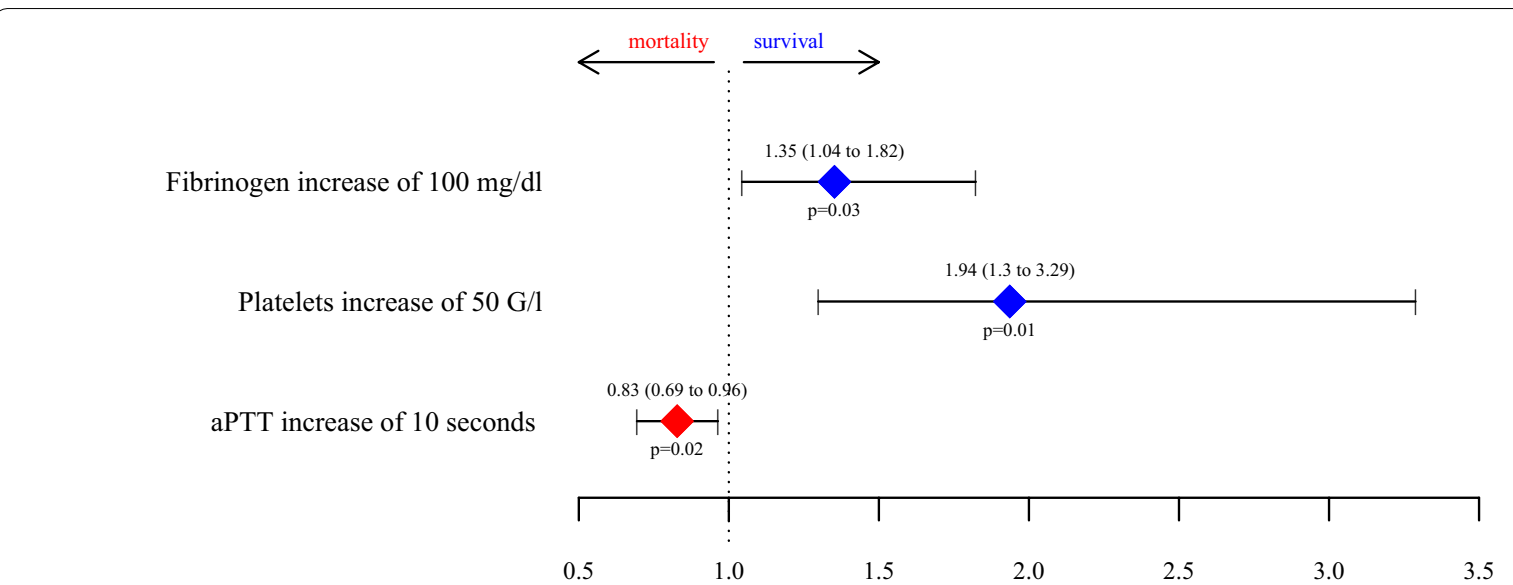

Fig. 3 Adjusted odds ratios $(95 \% \mathrm{Cl})$ for survival retrieved from logistic regression. For an odds ratio greater than 1, an increase in the parameter decreases the mortality risk

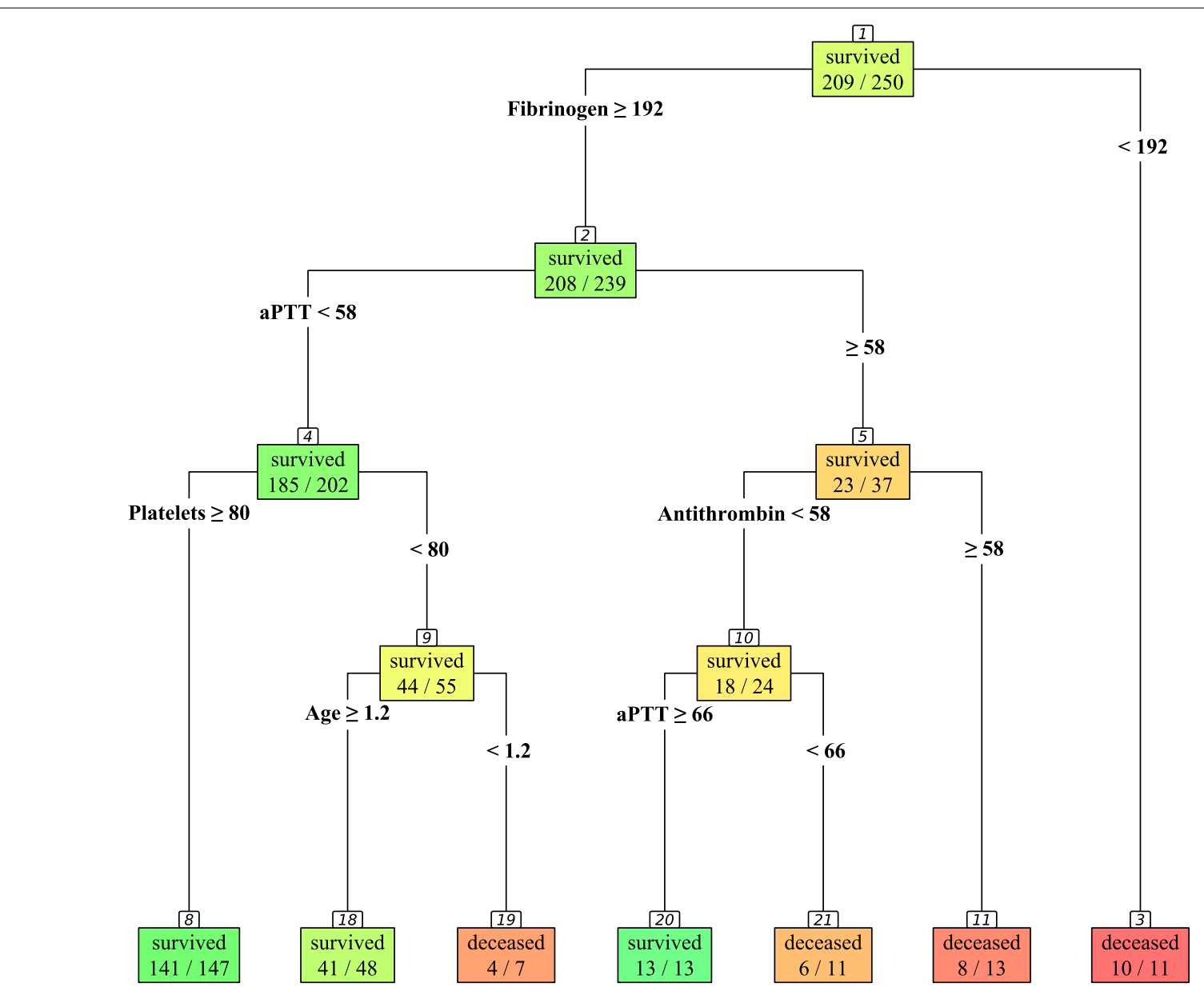

Fig. 4 Decision tree for survival. The classification tree was fitted with univariate predictors at the peak of $\mathrm{C}$-reactive protein and age 


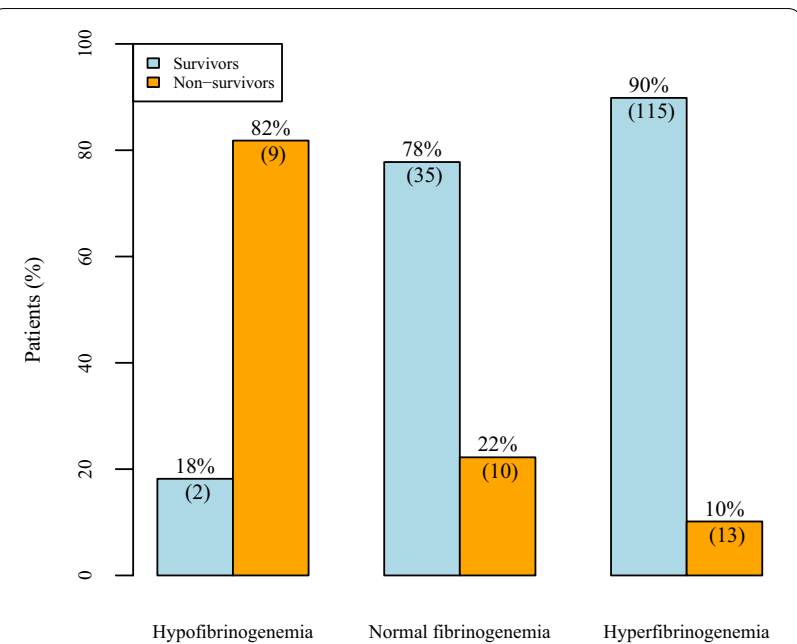

Fig. 5 Survival by age-adjusted hypofibrinogenemia, normal fibrinogenemia and hyperfibrinogenemia. The classification is based on the age-adjusted norm value ranges

to poor outcome $[4,46]$. Especially in neonates, isolated thrombocytopenia leads to increased mortality. This may be explained by the fact that during the first 6 weeks after birth, the plasmatic coagulation cascade is not fully developed and therefore hemostatic competence heavily relies on platelet function [47].

Overall, a decreased platelet count is associated with mortality. This might be due to the fact that thrombocytes are also an important part of the innate immune system. Whenever pathogens are present, platelets bind to them via the glycoprotein receptor IIb/IIIa and Toll-like receptors. That either happens directly, or it is mediated by plasma proteins like fibrinogen [48]. The activated platelets release antimicrobial peptides like defensins, kinocidins and thrombocidins to kill bacteria. In addition, they release members of the chemokine family like platelet factor 4 (PF4, CXCL-4) in order to attract other immune cells $[49,50]$.

The third coagulation parameter essential for the prediction of mortality in septic children in our study was aPTT. Prolongation leads to poorer outcome. A long aPTT might reflect the most severe cases of sepsis due to consumption of coagulation factors or highdose heparin therapy, but may also be caused by a FXII deficiency. In septic patients, FXII deficiency can be protective via attenuation of the FXII-dependent bradykinin generation, complement activation and further contact pathway activation. This consequence of low FXII levels might explain why $13 / 13$ children with fibrinogen levels above $192 \mathrm{mg} / \mathrm{dl}$ and an aPTT above 66 s survived, despite an antithrombin level below 58 .
Compared with developed countries, at $16.4 \%$ the overall mortality of our children was in the upper frame [51, 52]. Due to the tertiary care status of our university hospital, the study included a large number of seriously ill children. Of them, 20.4\% developed septic shock with a mortality rate of $41.2 \%$. The literature reports a mortality rate of $20-30 \%$ in the presence of septic shock [53, 54], which increases to $52 \%$ in the case of additional MODS.

The limitations of our study have to be mentioned. The retrospective design of the study precludes assessment of causal relationships. Nevertheless, the main finding that high fibrinogen levels are beneficial in sepsis can be well explained by recently reported pathophysiological mechanisms. Further, not all the considered parameters were available throughout the observation period of 7 days. Especially for children who died rapidly or were transferred to another hospital soon after C-reactive protein peaked, data are lacking and a bias could be present in the consecutive days due to the smaller sample size. Therefore, the main analyses were performed solely on the day of $\mathrm{C}$-reactive protein peak. The number of 250 sepsis cases diagnosed in children over a period of 14 years could be considered low. A deeper insight might be gained in a prospective setting.

Interpretation of the results of the multivariate logistic regression analysis for survival and decision tree analysis should be done carefully, since it cannot be concluded that these coagulation parameters are independently associated with survival because major well-known risk factors for death such as organ dysfunction or lactate were not taken into account. These analyses were used solely in an explorative fashion to describe the relation of the inflammatory and coagulatory parameters to survival. Therefore, this study is mainly descriptive and cannot determine the predictive value of coagulation parameters for outcome after sepsis. Another limitation is the fact that some values, such as low fibrinogen or low platelets, might have led to interventions that were not captured by this retrospective study. This could have influenced the magnitude of the associations. Despite all limitations, this study gives new insight into the course of sepsis and provides a good basis for a prospective study using these parameters as proxy for mortality, like organ dysfunction resolution or new and progressive MODS.

\section{Conclusion}

The link between inflammation and coagulation plays a crucial role in children with sepsis. C-reactive protein does not allow discrimination between survivors and non-survivors. In contrast, increased levels of fibrinogen and platelets are linked to survival and distinctively reflect the inflammatory process. Prolonged 
aPTT is associated with lower survival, which might reflect therapy-related measures needed due to disease severity.

\section{Additional files}

Additional file 1. Receiver operating characteristic (ROC) curves and area under the ROC curve (AUC) values for survival. The ROC curves showing the predictive value of fibrinogen. (A) Platelets (B) and aPTT (C). ROC AUC is provided with $95 \% \mathrm{Cls}$.

Additional file 2. Sensitivity and specificity analysis for mortality of fibrinogen, platelets and aPTT.

Additional file 3. Fibrinogen (Clauss method) norm value ranges for female and male children.

\section{Abbreviations}

AIC: Akaike information criterion; AMP: antimicrobial peptides; aPTT: activated partial thromboplastin time; CRP: C-reactive protein; DIC: disseminated intravascular coagulopathy; FXII: coagulation factor XII; GAS: group A Streptococci; GBS: group B Streptococci; ICD: International Statistical Classification of Diseases and Related Health Problems; MODS: multiple organ dysfunction syndrome; OR: odds ratio; PF4: platelet factor 4; PICU: pediatric intensive care unit; PT: prothrombin time.

\section{Authors' contributions}

CN conceived the study, developed the study design, contributed to the acquisition and interpretation of data and drafted and edited the manuscript. $\mathrm{MB}$ conceived the study, developed the study design and contributed to the acquisition and interpretation of data. She also drafted and edited the manuscript. She was responsible for ensuring that this study was reported honestly, accurately and transparently and also for the overall work by ensuring that questions pertaining to the accuracy or integrity of any portion of the work were appropriately investigated and resolved (corresponding author). TH performed the statistical analysis and gave support in interpretation of the data. He also contributed significantly to the drafting and editing the manuscript. CL gave support in development of the study design and contributed to the statistical analysis. She contributed to the drafting and editing the manuscript. AE contributed to the interpretation of data. He also gave support in drafting and editing the manuscript. AB helped with the English translation and assisted with drafting and editing the manuscript. KA contributed to the acquisition and interpretation of data. She also assisted with drafting and editing the manuscript. KA contributed to the acquisition and interpretation of data. She also assisted with drafting and editing the manuscript. PI contributed to the interpretation of data and gave major support in drafting and editing the manuscript. All authors read and approved the final manuscript.

\section{Author details}

1 Department of Pediatrics, Pediatrics I, Medical University of Innsbruck, Anichstrasse 35, 6020 Innsbruck, Austria. ${ }^{2}$ Institute for Sports Medicine, Alpine Medicine and Health Tourism, UMIT - University for Health Sciences, Medical Informatics and Technology, Eduard Wallnöfer Zentrum 1, 6060 Hall in Tirol, Austria. ${ }^{3}$ Department of Mathematics, Faculty of Mathematics, Computer Science and Physics, University of Innsbruck, Technikerstraße 13, 6020 Innsbruck, Austria. ${ }^{4}$ Department of Medical Statistics, Informatics and Health Economics, Medical University of Innsbruck, Anichstrasse 35, 6020 Innsbruck, Austria. ${ }^{5}$ Department of General and Surgical Critical Care Medicine, Medical University of Innsbruck, Anichstrasse 35, 6020 Innsbruck, Austria. ${ }^{6}$ Department of Anaesthesiology and Critical Care Medicine, Medical University of Innsbruck, Anichstrasse 35, 6020 Innsbruck, Austria.

\section{Acknowledgements}

We thank Dr. Sophie Hofer, Dr. Christina Schoner, Dr. Daniela Hainz, Dr. Ahmed Fideh, Martin Wagner, Dr. Thomas Varga and Dr. Dietmar Fries for their assistance in executing this project.

\section{Competing interests}

The authors state that they have no competing interests in regard to this study. MB has received personal fees from LFB Biomedicaments, Baxter $\mathrm{GmbH}$, CSL Behring $\mathrm{GmbH}$ and Mitsubishi Tanabe and non-financial support from TEM International outside the submitted work. PI has received personal fees from Baxter $\mathrm{GmbH}$, personal fees from CSL Behring $\mathrm{GmbH}$, personal fees from Fresenius Kabi GmbH Austria, personal fees from Bayer GmbH Austria and personal fees from LFB and non-financial support from TEM International outside the submitted work.

\section{Availability of data and materials}

All datasets used and/or analyzed during the current study are available from the corresponding author upon reasonable request.

\section{Consent for publication}

All authors consent to publication.

\section{Ethics approval and consent to participate}

The study was approved by the Ethics Committee of the Medical University of Innsbruck. It was not necessary to obtain written informed consent since the data were anonymized.

\section{Funding}

There is no funding to declare.

\section{Publisher's Note}

Springer Nature remains neutral with regard to jurisdictional claims in published maps and institutional affiliations.

Received: 19 March 2018 Accepted: 9 November 2018

Published online: 16 November 2018

\section{References}

1. Goldstein B, Giroir B, Randolph A. International pediatric sepsis consensus conference: definitions for sepsis and organ dysfunction in pediatrics. Pediatr Crit Care Med. 2005;6(1):2-8.

2. Randolph AG, McCulloh RJ. Pediatric sepsis: important considerations for diagnosing and managing severe infections in infants, children, and adolescents. Virulence. 2014;5(1):179-89.

3. Hartman ME, Linde-Zwirble WT, Angus DC, Watson RS. Trends in the epidemiology of pediatric severe sepsis*. Pediatr Crit Care Med. 2013;14(7):686-93.

4. Sokou R, Giallouros G, Konstantinidi A, Pantavou K, Nikolopoulos $\mathrm{G}$, Bonovas $\mathrm{S}$, et al. Thromboelastometry for diagnosis of neonatal sepsis-associated coagulopathy: an observational study. Eur J Pediatr. 2018;177(3):355-62.

5. van der Poll T, van Deventer SJ. Cytokines and anticytokines in the pathogenesis of sepsis. Infect Dis Clin North Am. 1999;13(2):413-26.

6. Reddy RC, Chen GH, Tekchandani PK, Standiford TJ. Sepsis-induced immunosuppression. Immunol Res. 2001;24(3):273-87.

7. Levy MM, Fink MP, Marshall JC, Abraham E, Angus D, Cook D, et al. 2001 SCCM/ESICM/ACCP/ATS/SIS international sepsis definitions conference. Crit Care Med. 2003:31(4):1250-6.

8. Schneider HG, Lam QT. Procalcitonin for the clinical laboratory: a review. Pathology. 2007:39(4):383-90.

9. Castelli GP, Pognani C, Meisner M, Stuani A, Bellomi D, Sgarbi L. Procalcitonin and $C$-reactive protein during systemic inflammatory response syndrome, sepsis and organ dysfunction. Crit Care. 2004;8(4):R234-42.

10. Clec'h C, Ferriere F, Karoubi P, Fosse JP, Cupa M, Hoang P, et al. Diagnostic and prognostic value of procalcitonin in patients with septic shock. Crit Care Med. 2004;32(5):1166-9.

11. Chiesa C, Natale F, Pascone R, Osborn JF, Pacifico L, Bonci E, et al. C reactive protein and procalcitonin: reference intervals for preterm and term newborns during the early neonatal period. Clin Chim Acta. 2011:412(11):1053-9.

12. Schentag JJ, O'Keeffe D, Marmion M, Wels PB. C-reactive protein as an indicator of infection relapse in patients with abdominal sepsis. Arch Surg. 1984;119(3):300-4. 
13. Maury CP. Monitoring the acute phase response: comparison of tumour necrosis factor (cachectin) and C-reactive protein responses in inflammatory and infectious diseases. J Clin Pathol. 1989;42(10):1078-82.

14. Povoa P, Almeida E, Moreira P, Fernandes A, Mealha R, Aragao A, et al. C-reactive protein as an indicator of sepsis. Intensive Care Med. 1998;24(10):1052-6.

15. Presterl E, Staudinger T, Pettermann M, Lassnigg A, Burgmann $H$, Winkler S, et al. Cytokine profile and correlation to the APACHE III and MPM II scores in patients with sepsis. Am J Respir Crit Care Med. 1997;156(3 Pt 1):825-32.

16. Yentis SM, Soni N, Sheldon J. C-reactive protein as an indicator of resolution of sepsis in the intensive care unit. Intensive Care Med. 1995;21(7):602-5.

17. Pinilla JC, Hayes P, Laverty W, Arnold C, Laxdal V. The C-reactive protein to prealbumin ratio correlates with the severity of multiple organ dysfunction. Surgery. 1998;124(4):799-805 (discussion-6).

18. Waydhas C, Nast-Kolb D, Trupka A, Zettl R, Kick M, Wiesholler J, et al. Posttraumatic inflammatory response, secondary operations, and late multiple organ failure. J Trauma. 1996;40(4):624-30 (discussion 630-1).

19. Ikei S, Ogawa M, Yamaguchi Y. Blood concentrations of polymorphonuclear leucocyte elastase and interleukin-6 are indicators for the occurrence of multiple organ failures at the early stage of acute pancreatitis. J Gastroenterol Hepatol. 1998;13(12):1274-83.

20. de Beaux AC, Goldie AS, Ross JA, Carter DC, Fearon KC. Serum concentrations of inflammatory mediators related to organ failure in patients with acute pancreatitis. Br J Surg. 1996:83(3):349-53.

21. Rau B, Steinbach G, Baumgart K, Gansauge F, Grunert A, Beger HG. Serum amyloid A versus C-reactive protein in acute pancreatitis: clinical value of an alternative acute-phase reactant. Crit Care Med. 2000;28(3):736-42.

22. Levi M, van der Poll T. Inflammation and coagulation. Crit Care Med. 2010;38(2 Suppl):S26-34.

23. van der Poll T, Levi M. Crosstalk between inflammation and coagulation: the lessons of sepsis. Curr Vasc Pharmacol. 2012;10(5):632-8.

24. Berends ET, Kuipers A, Ravesloot MM, Urbanus RT, Rooijakkers SH. Bacteria under stress by complement and coagulation. FEMS Microbiol Rev. 2014;38(6):1146-71

25. Pahlman LI, Morgelin M, Kasetty G, Olin Al, Schmidtchen A, Herwald H. Antimicrobial activity of fibrinogen and fibrinogen-derived peptides-a novel link between coagulation and innate immunity. Thromb Haemost. 2013;109(5):930-9.

26. Senior RM, Skogen WF, Griffin GL, Wilner GD. Effects of fibrinogen derivatives upon the inflammatory response. Studies with human fibrinopeptide B. J Clin Invest. 1986;77(3):1014-9.

27. Lauterbach R, Pawlik D, Radziszewska R, Wozniak J, Rytlewski K. Plasma antithrombin III and protein C levels in early recognition of late-onset sepsis in newborns. Eur J Pediatr. 2006;165(9):585-9.

28. Semeraro F, Colucci M, Caironi P, Masson S, Ammollo CT, Teli R, et al. Platelet drop and fibrinolytic shutdown in patients with sepsis. Crit Care Med. 2018:46(3):e221-8.

29. Esmon CT. The interactions between inflammation and coagulation. $\mathrm{Br}$ J Haematol. 2005;131(4):417-30.

30. Akaike H. A new look at the statistical model identification. IEEE Trans Autom Control. 1974;19(6):716-23.

31. Ersoy B, Nehir H, Altinoz S, Yilmaz O, Dundar PE, Aydogan A. Prognostic value of initial antithrombin levels in neonatal sepsis. Indian Pediatr. 2007;44(8):581-4

32. Taylor FB Jr, Kinasewitz GT, Lupu F. Pathophysiology, staging and therapy of severe sepsis in baboon models. J Cell Mol Med. 2012;16(4):672-82.

33. Luo D, Szaba FM, Kummer LW, Plow EF, Mackman N, Gailani D, et al. Protective roles for fibrin, tissue factor, plasminogen activator inhibitor-1, and thrombin activatable fibrinolysis inhibitor, but not factor XI, during defense against the gram-negative bacterium Yersinia enterocolitica. J Immunol. 2011;187(4):1866-76.
34. Mullarky IK, Szaba FM, Berggren KN, Parent MA, Kummer LW, Chen W, et al. Infection-stimulated fibrin deposition controls hemorrhage and limits hepatic bacterial growth during listeriosis. Infect Immun. 2005;73(7):3888-95.

35. Davalos D, Akassoglou K. Fibrinogen as a key regulator of inflammation in disease. Semin Immunopathol. 2012;34(1):43-62.

36. McAdow M, Missiakas DM, Schneewind O. Staphylococcus aureus secretes coagulase and von Willebrand factor binding protein to modify the coagulation cascade and establish host infections. J Innate Immun. 2012;4(2):141-8.

37. Jennewein C, Mehring M, Tran N, Paulus P, Ockelmann PA, Habeck K, et al. The fibrinopeptide bbeta15-42 reduces inflammation in mice subjected to polymicrobial sepsis. Shock. 2012;38(3):275-80.

38. Wolf T, Kann G, Becker S, Stephan C, Brodt HR, de Leuw P, et al. Severe Ebola virus disease with vascular leakage and multiorgan failure: treatment of a patient in intensive care. Lancet. 2015;385(9976):1428-35.

39. Wada H, Mori Y, Okabayashi K, Gabazza EC, Kushiya F, Watanabe M, et al. High plasma fibrinogen level is associated with poor clinical outcome in DIC patients. Am J Hematol. 2003;72(1):1-7.

40. Renckens R, Roelofs JJ, Stegenga ME, Florquin S, Levi M, Carmeliet P, et al. Transgenic tissue-type plasminogen activator expression improves host defense during Klebsiella pneumonia. J Thromb Haemost. 2008;6(4):660-8.

41. Asakura H, Ontachi Y, Mizutani T, Kato M, Saito M, Kumabashiri I, et al. An enhanced fibrinolysis prevents the development of multiple organ failure in disseminated intravascular coagulation in spite of much activation of blood coagulation. Crit Care Med. 2001;29(6):1164-8.

42. Mihajlovic D, Lendak D, Mitic G, Cebovic T, Draskovic B, Novakov A, et al. Prognostic value of hemostasis-related parameters for prediction of organ dysfunction and mortality in sepsis. Turk J Med Sci. 2015;45(1):93-8.

43. Semeraro N, Ammollo CT, Semeraro F, Colucci M. Coagulopathy of acute sepsis. Semin Thromb Hemost. 2015;41(6):650-8.

44. Parker RI. Coagulopathies in the PICU: DIC and liver disease. Crit Care Clin. 2013;29(2):319-33.

45. Nesseler N, Launey Y, Aninat C, Morel F, Malledant Y, Seguin P. Clinical review: the liver in sepsis. Crit Care. 2012;16(5):235.

46. Ostrowski SR, Haase N, Muller RB, Moller MH, Pott FC, Perner A, et al. Association between biomarkers of endothelial injury and hypocoagulability in patients with severe sepsis: a prospective study. Crit Care. 2015;19(191):015-0918.

47. Andrew M, Vegh P, Johnston M, Bowker J, Ofosu F, Mitchell L. Maturation of the hemostatic system during childhood. Blood. 1992;80(8):1998-2005.

48. Yeaman MR. Platelets in defense against bacterial pathogens. Cell Mol Life Sci. 2010;67(4):525-44.

49. Clemetson KJ. The role of platelets in defence against pathogens. Hamostaseologie. 2011;31(4):264-8.

50. Saloga J, Klimek L, Buhl R, Mann W, Knop J, Grabbe S. Allergologie-Handbuch: Grundlagen und klinische Praxis. Stuttgart: Schattauer Verlag; 2012.

51. Taori RN, Lahiri KR, Tullu MS. Performance of PRISM (pediatric risk of mortality) score and PIM (pediatric index of mortality) score in a tertiary care pediatric ICU. Indian J Pediatr. 2010;77(3):267-71.

52. Kutko MC, Calarco MP, Flaherty MB, Helmrich RF, Ushay HM, Pon S, et al. Mortality rates in pediatric septic shock with and without multiple organ system failure. Pediatr Crit Care Med. 2003;4(3):333-7.

53. Hatherill M, Tibby SM, Turner C, Ratnavel N, Murdoch IA. Procalcitonin and cytokine levels: relationship to organ failure and mortality in pediatric septic shock. Crit Care Med. 2000;28(7):2591-4.

54. Wilkinson JD, Pollack MM, Ruttimann UE, Glass NL, Yeh TS. Outcome of pediatric patients with multiple organ system failure. Crit Care Med. 1986:14(4):271-4. 